5. I. LIBRARY 







\section{FIRST STPPLWMIENT}

\section{TO THE}

\section{LIST OF COLEOPTREA: \\ O F}

\section{AMERICA, NORTH OF MEXICO.}

\section{BY SAMUEL HENSHAW.}

On the following pages I give such corrections and additions to the List of Coleoptera, published by the American Entomological Society in October I 885, as have come to my notice. So far as I know it includes all additions published up to January ist I 887.

The authority is given for new genera, the number in brackets indicating their position in the List.

The new species are numbered continuously with the List.

CARABIDAE.

Notiophilus.

9239. aquaticus $\operatorname{Linn}$.

Nebria.

9240. frigida Sahlb.

924I. parvula Sahlb.

\section{Bembidium.}

360. = scintillans Bates.

392. $\|=$ flavopictum Mots.

Pterostichus.

562. $\|=$ cyanicolor Chd. 9242. splendida Sahlb.

9243. epipleuralis Sahlb. 9244. plana Sahlb.

\section{Amara.}

9245. angustata \| Sahlb.
Dicaelus.

Flohri Bates. $=713$.

Anchonoderus,

844. $=$ myops Reiche.

Galerita.

$850 .=$ mexicana chd.

Blechrus.

9I . $=$ ? glabratus Dufts.

Chlaenius.

1036. = caeruleicollis Chd.

ro33. $f=$ chrysopleuralis chd.

Anisodactylus.

Irg2. = mexicanus $D e j$.

9246. brevicollis Chd. 


\section{DYTISCIDAE.}

Agabus.

1432. = mutus Sharp.

GYRINIDAE。

Gyrinus.

$1526 .=1521$.

\section{HYDROPHILIDAE.}

Hydrophilus.

9247. ater Fab.

${ }_{1588}-1595$ belong to

Tropisternus Sol. ( $15^{8} 7$ ).

9348. apicipalpis Chev.

\section{Limnebius.}

9249. polita Casey. 9250. angustula Casey. 925 I. alutacea Casey. 9252. congener Casey. 9253. coniciventris Casey.

\section{SILPHIDAE.}

Silpha.

1711, ramosa Say. aenescens Casey.

\section{SCYDMAENIDAE}

Scydmaenus.

I840. $\|=$ Lecontei Sehantî.
Cholerus.

$1853=1854$.

\section{PSELAPHIDAE.}

\section{Batrisus}

9254. mendocino Casey. 9255. zephyrinus Casey. 13 h 9256. speculum Casey. 9257. monticola Casey. 9258. occiduus Casey. I914-I916 belong to Rybaxis Saulcy. (I9I3).

Bryaxis. 9259. texana Casey. 9260. infinita Casey. 1926-1927 belong to Nisa Casey. (1925).

Reichenbachia Leach. (1946). 9261. tumorosa Casey. 9262. tumidicornis Casey. 9263. informis Casey.
9264. gracilicornis Casey. 9265. nevadensis Casey. 9266. fundata Casey. 9267. franciscana Casey. 1947-1948 belong to Nisaxis Casey. (1946). I953 - I955 belong to Sonoma Casey. (1952). Faronus $\ddagger$ Lec. I 956 belongs to Oropus, Casey. (1955).

Trichonya $\ddagger$ Lec. 9268. convexus Casey. 9269, interruptus Casey. 9270. abbreviatus Casey.

Trimium.

Actium Casey. 


\section{STAPHYLINIDAE,}

\section{Falagria.}

9271. occidua Casey.

9272. laticollis Casey.

Colusa Casey. (2004). 9273. gracilis Casey. 9274. eximia Casey. 9275. valida Casey. 9276. exilis Casey. 9277. grandicollis Casey.

; 2007 belongs to

Pontomalota Casey. (2006). 9278. californica Casey. 9279. nigriceps Casey.

\section{Lomechusa.} 9280. montana Casey.

\section{Tachyusa.} 9281. experta Casey. 9282. linearis Casey. 9283. laticeps Casey. 9284. faceta Casey. 9285. Harfordi Casey. 9.286. crebrepunctata Casey.

\section{Platyusa.} 9287. sonomae Casey.

\section{Calodera.} 9288. attenuata Casey.

\section{Maseochara.} 9289. californica Casey.

\section{Dasyglossa.} 9290. prospera $\mathrm{Er}$.

\section{Oxypoda.}

1 929I. insignis Casey.

\section{Autalia.} 9292. elegans Casey.

\section{Phytosus.}

9293. bicolor Casey. 9294. maritimus Casey.

\section{Bolitochara.}

9295. californica Casey. 9296. nigrina Casey.
Heterothops.

9297. occidentis Casey. exilis || Casey,

Staphylinus. $214 \mathrm{I}=$ =viridanus $H_{0} \mathrm{~m}$.

Belonuchus. 9298. pallens Sharp.

Philonthus. 9299. quisqniliarius Gyll.

Cafius Steph. Bryonomus Casey.

\section{Stenus.}

2342. =scabiosus Casey. 2366.=pumilio $E r$. 2377.=humilis $\mathrm{Er}$. 2406-=morio Grav. 2449.=tarsalis Ljungh. 9300. jejunus Casey. 9301. sibiricus Sahlb.

\section{Cryptobium.}

Hesperobium Casey.

\section{Ababactus.}

9302. pallidiceps Casey.

2560 belongs to

Trachysectus Casey. (2543).

Caloderma Casey. (2543). 9303. rugosum Casey. 9304. continens Casey. 9305. angulatum Casey. 9306. mobile Casey. 9307. contractum Casey. 9308. luculentum Casey. 9309. reductum Casey. 9310. tantillum Casey.

Oligopterus Casey. (2543). 93II. cuneicollis Casey.

Melon Steph. (2543). 93 I2. sinuatocollis Casey. 9313. convergens Casey. 9314. lepida Casey. 9315. puberula Casey. 
9316. malaca Casey.

9317. latiuscula Casey.

9318. sublesta Casey.

9319. consanguinea Casey.

9320. contigua Casey.

9321. luctuosa Cisey.

9322. retrusa Casey.

9323. gregalis Casey.

9324. mimula Casey.

9325. languida Ca $e \%$.

\section{Lithocharis.}

Metaxyodonta Casey. 9326. alutacea $C$ isey. 9327. quadricollis Ca.ey.

Lena Casey. (2564) 9328. testacea $C i s y$. 2559 and 2673 belong to

Aderocharis Sharp. (2566).

Ramona Casèy. 2566. 9329. capitulum $\mathrm{Ca}$ ey.

Leptogenius Casey. ${ }^{25} 8_{3}$ ). 9330. brevicomis Casey.

\section{Scopaeus.}

933I. rotundiceps Casey.

9332. truncaticeps Casey.

2545 belongs to

Scopaeodera Casej. (2549).

2546-47 belong to

Leptorus Casey. (2549).

9333. texanus Cas y.

9334. bicolor Casey.

9335. versicolor Casey.

9336. longiceps Casey.

2550 belongs to

Orus Casey, (2549).

9337. parallelus Casey.

Tachinus.

9338. arcticus Mots.

\section{Habrocerus.}

Eumitocerus Casey. 9339. tarsalis Casey.

\section{Platystethus.}

9340. spiculus $E r$.

\section{A pocellus.}

9341. niger Casey.

Phloeopterus Mots. (2802).

9342. filicornis Casey.

9343. longipalpus Casey.

Vellica Casey. (2802).

9344. longipennis Casey.

\section{Lesteva.}

9345. truncata Casey.

\section{Amphichroum.}

9346. flavicorne Casey.

9347. alutaceum Casey.

9348. pilosellum Casey.

9349. veterator Casey.

9350. crassicorne Casey.

Pelecomalium Casey. (28I9). 935 I. binotatum Casey. 9352. modestum Casey.

\section{Lathrimaeum.}

9353. humerale Casey.

\section{Homalium.}

9354. algarum Casey. fucicola || Casey.

9355. rugipenne Casey.

\section{Orobanus.}

9356. densus Casey.

9357. rufipes Casey.

\section{Protinus.}

9358. salebrosus Casey.

\section{TRICHOPTERYGIDAE.}

\section{Actidium.}

9359. rotundicolle Casey. 9360, robustulum Cis y'. 9361. granulosu n Cresey. 9362. attenuatum Casey.

\section{Ptilium.}

9363. sulcatum Casey.
Trichopteryx.

9364. Crotchii Math.

\section{Smicrus.}

9365. americanus Caser.

Ptinella,

9366. ? nigrovittis Lee. 
SCAPHIDIIDAE.

2979 belungs to

Baeocera.

CORYLOPHIDAE. 3023-3024 belong to Corylophodes Matth.

COCCINELLIDAE.

Exochomus.

septentrionis Weise $=3 n 8_{3}$.

CRYPTOPHAGIDAE.

Cryptophagus.

9367. beringensis Sahll.

DERMESTIDAE.

Attagenus.

9368. varicolor Jifyne.

\section{NITIDULIDAE.}

Pallodes.

3747. = pallidus Beauv.

\section{LATRIDIIDAE.}

Cartodera Thoms. (3796) 9369. intermedia Belon.

\section{Corticaria:}

$3797=$ pubescens Ifummel.

\section{Byrrhus.}

9371. fasciatus kibb.
3804. = fenestralis Lin. 3823. = distinguenda $\mathrm{Com}$. 9370. fulva Com.

BYRRHIDAE.

Ditaphrus Casey. (3910). 9372. scymnoides Caser'.

DASCYLLIDAE.

Euscaphurus Casey. (3987).

9373. saltator Casey.

\section{ELATERIDAE.}

Melasis.

9374. rufipennis Hom.

\section{Stethon.}

9375. errans Horn.

Eucriemis Ahrens. (4029). 9376. americanus Horn. 4046 belongs to

Deltometopus.

Dromaeolus.

9.77. nitens Horn.

9378. Harringtoni Horn. 9379 pusillus Horn.

Fornax. 9380 . infelix 11 orn.
Microrrhagus.

938r. audax Horn. 9382. Bonvouloiri Horm.

Nematodes.

9383. pavidus $/$ torm. 9384. teres $M$ Iorn.

Phlegon.

9385. Ulkei Horn.

\section{Cryptohypnus.}

9386. barbatus Sulill.

Elater.

- ? fuscatus 1 Kels $/ \mathrm{t}=4235$.

- ? semivitatus Sor. $=4483$. 


\section{$-2 \times 8-$ \\ BUPRESTIDAE.}

Buprestis.

46ro. = aurulenta Limm.

9387 villosa $L e c$.

Chrysobothris.

$4639 \mathrm{f} .=4642$.

$4649=4648$.

$4653 \mathrm{a}=4653$.

$4655=9405$.

- cribraria Mann $=4639$.

- dissimilis Gory $=4639$.

- posticalis $L$. d $G=4647$.

- subcylindrica Mots $=4654$ -

$\angle-$ errans Gory. $=$ S. Amer.

_- frontalis Oliv.-S. Amer.

- thoracica $F a b=I V$. I.

9388: Edwardsii Horn.

9389. axillaris Hon?

9390. pirpureovittata Home.

9391. speculifer Hom?.

9392. viridicyanea Horn.

9393. dolata IIorn.

9394. ludificata Hom.
9395. caurina Horr.

9396. Blanchardi Hom?.

9397. mali Horn.

9398. nixa Horn.

9399. deserta Horr.

9400. lixa Horn.

9401. cyanella Horn.

9402. humilis Horn.

9403. aerea Chev.

9404. Merkelii Horn.

9405. acutipennis Chev.

9406. libonoti Horn:

9407. impressa Fab.

9408. chalcophoroides ILon $r_{\text {. }}$

9409. scitula Gory.

9410. prasina Horn.

Belionota.

- californica Mots $=4666$.

\section{Polycesta.}

94II. angulosa Duval.

MALACHIDAE.

509 I belongs to

Listrus.

PTINIDAE.

\section{Caenocara.}

941.2. occidens Gerey',

Dinapate Horn. (5353).

9413. Wrightii Horm.

\section{LUOCANIDAE.}

Platycerus.

9414. californicus Casey. 1 ।

\section{SCARABAEIDAE.}

\section{Bradycínetus}

9415. Hornii Rivers.

Malacopterus.

sor6 = lineatus Guer,

\section{Toxotus.}

9417. nubifer Lec.

Leptura.

63I9 $=$ nitens Forst.

$6325 .=$ var, of 6323 .
Thyce.

9416. marginata Casey.

\section{CERAMBYCIDAE.}

$6365 .=6265$.

9418. vexatrix Mann.

6432 belorigs to

Nossodrys Bater. (6448).

contempta Bates $=643$ ?.

Tetraopes.

6509, = rubrocinereus Thoms. 
Saxinis.

9419. propinqua Jac.

Fidia.

9420. lurida Lefr.

Adoxus.

6726 = obscurus Line.

Myochrous.

- ? plagiata Melsh $=672 \pi$.

Tymnes.

picipes Oliv. $=9427$.

9421. chrysis Oliv.

Paria.

6747. = canella Fub.

- thoracica Melsh $=6748$. melanura Helsh. $=6748$.

\section{Metachroma.}

6757. interrupta say. ? histrio Lefr.

6760. = lurida Oliv.

- cuprea Prow. $=677 \mathrm{I}$.

\section{Graphops.}

9422. beryllinus Lee.

9423. obscurus Lec.

9424. varians $L e c$.

9425. bicolor Lefr.

\section{Colaspis.}

6774. = suilla Fub.

$6774 \mathrm{~b} .=$ Crotchii Letr.

$6775 .=943^{\circ}$.

--. chrysis Oliv $=9424$.

9426. flavicans Lefir.

6778 belongs to

Nodonota Lefr. (6777).

strigicollis Lef $r:=6778 \mathrm{~b}$.

\section{CHRYSOMELIDAE.}

Rhabdopterus Lefr. $(6778)$.

9427, picipes Oliv.

Prasocuris.

6783. = vittata Oliv.

Doryphora,

${ }_{67} S_{6}=67 S_{3}$.

9428. Behrensi Har.

Phyllodecta.

9429. scutellaris Sahll.

Phyllobrotica.

685 I. = livida Lee.

Luperus.

9430: maculicollis Ler:

943I. torquatus Lee.

Adimonia.

6902. = femoralis Melsh.

Hypolampsis.

$$
\text { rugosa oliv: }=6020 \text {. }
$$

Oedionychis. 9432. Horni Har.

\section{Disonycha.}

6949. = caroliniana Frib.

\section{Haltica.}

$6964 .=$ var. of 6963.

- kalmiae Melsh. $=6963$.

9433. polita Oliv.

9434. janthina Lee.

Lactica.

-6975. = tibialis Olic. 6976. = iris Oliv.

Crepidodera. - iris Oliv. $=6976$.

\section{Chaetocnema.}

Lecontei $D_{u}=7039$.

\section{BRUCHIDAE}

Bruchus

longiventras Sharp: $=7139$.
Zabrotes.

9435. subnitens $/ /(n)$.

TENEBR:ONIDAE.

Eleates Casey. (7542).

9436. occidentalis Casey.

\section{CISTELIDAE.}

Cistela.

pulla Melsh $=7607$. 
OTHNIIDAE.

Othnius.

9437. guttulatus Lec.

$-220-$

MELANDRYIDAE.

Dircaea. $=$

Phloeotrya Steph.

$767 \mathrm{I}=$ =Vaudoueri Mluls

PYTHIDAE.

Pytho.

7710. = planus Oliv.

\section{MORDELLIDAE.}

Mordella.

atrata $M$ Melsh $=7780$.

dimidiata Melsh.=European.

modesta Melsh: $=785 \mathrm{I}$.

pubescens $F a b .=785$ I.

943 . immaculata Smith.

9439. flaviventris Smith.
9443. carinata Smitr.

Mordellistena.

9441. erratica Smith.

9442. tarsalis Smith.

9443. pratensis Smith.

9444. conformis Smith.

9445. hirticula Smith.

\section{MELOIDAE.}

Macrobasis.

$8060 .=$ torsa Lec.

\section{OTIORHYNCHIDAE}

Otiorhynchus.

9446. picipes $F a b$.

CURCULIONDAE.

Barinus Casey. (8949). 9447. squamolineatus Casey.

CALANDRIDAE.

Spherophorus.

fasciatus $F u b$. Not N. Amer.
9047. belongs to

Allomimus.

\section{SCOLYTIDAE.}

- yo59-9o6r belong to

Gnathotrichus.

Pityophthorus.

9448. querciperda $S z$.

9102 belongs to

Cryphalus.

Dryocoetes.

9116. = autographus Rutz.

Tomicus.

$9 \mathrm{r} 3 \mathrm{I}=9 \mathrm{ror}$.
Hylesinus.

9449. trifolii Nuell.

Bothrosternus.

9450. Hubbardi s'z.

Renocis Casey. (9175).

9451. heterodoxus Casey.

Crypturgus.

9186. = pusillus Gyll.

Hylurgops.

9 r.99. = glabratus Zett . 





SMITHSONIAN INSTITUTION LIBRARIES 